\title{
Transjugular Liver Biopsy: Results of 97 Patients
}

\author{
Halil Dönmez¹, Güven Kahriman¹, Nevzat Özcan¹, Ertuğrul Mavili', Kemal Deniz² \\ ${ }^{1}$ Department of Radiology, Faculty of Medicine, Erciyes University, Gevher Nesibe Hospital, Kayseri, Turkey \\ ${ }^{2}$ Department of Pathology, Faculty of Medicine, Erciyes University, Gevher Nesibe Hospital, Kayseri, Turkey
}

\begin{abstract}
Objective: To evaluate the feasibility and efficacy of transjugular liver biopsy in patients with contraindicated percutaneous biopsy.
\end{abstract}

Materials and Methods: Between June 2005 and April 2010, 97 patients who were admitted for transjugular liver biopsy were enrolled in this retrospective study. All liver biopsies were obtained using an 18 gauge Quick-Core liver biopsy set through the right hepatic vein via the internal jugular vein. Clinical indication, histopathological diagnosis, and complications were noted.

Results: Primary technical success was achieved in 93 (95.8\%) patients. Hepatic veins could not be catheterized and opacified in two patients and in the remaining two patients the veins could be opacified and catheterized but we were not able to pass the biopsy needle into the hepatic vein because of the acute angle between the inferior vena cava and hepatic veins. At least two specimens were obtained from each patient. The most frequent histopathological diagnosis was cirrhosis. A subcutaneous hematoma around the puncture side was encountered in one patient.

Conclusion: Transjugular liver biopsy is a feasible and effective alternative in patients with contraindication for percutaneous biopsy.

Key Words: Transjugular liver biopsy, liver biopsy, liver cirrhosis

Received: 05.12.2010 Accepted: 02.06.2011

\section{Introduction}

Percutaneous liver biopsy (PLB) is the standard method for the diagnosis and treatment planning of diffuse parenchymal liver disease (1-4). Percutaneous liver biopsy is not suitable for patients with coagulation a $\Delta$ bnormalities and thrombocytopenia. In this situation, transjugular liver biopsy (TJLB) is the alternative biopsy method. In the TJLB, the liver tissue obtained from the vascular system minimizes the risk of bleeding $(1,5)$. This biopsy technique also provides the opportunity for simultaneous hepatic vein pressure measurement and venographic examination. This study presents our experience with the Quick-Core biopsy set (Cook, Bloomington, IN) for TJLB in patients with contraindicated percutaneous liver biopsy.

\section{Material and Methods}

Between June 2005 and April 2010, a total of 97 patients (66 males, 31 females) with a mean age of $46.74 \pm 12.7$ years (range: 18-74 years) were included in this retrospective study. The study group consisted of patients who had contraindications for PLB because of coagulation abnormalities (PT $>16$ sec, INR $>1.2$, PTT $>40 \mathrm{sec}$ and thrombocyte count $<60.000 /$ $\mathrm{cm}^{3}$ ) with laboratory and clinical findings suggestive of diffuse liver disease. In 29 patients there was concomitant gross ascites confirmed by ultrasound. All patients were informed about the indications and possible risks of the procedure and written informed consent was obtained from each patient, receiving approval from the Institutional Review Board. The biopsies were performed in the angiography suite by two radiologists with the use of digital subtraction angiography (Philips Integris 5000, Philips Medical Systems, Netherlands) and an ultrasound (Toshiba Nemio 20, Toshiba Medical Systems, Japan) machine.

All patients lay in the supine position with a pillow placed under their shoulder. The procedure was performed under local anaesthesia with continuous monitoring.

The venous structures including the internal jugular vein (IJV), hepatic veins and inferior vena cava (IVC) were assessed by ultrasound prior to the procedure to confirm the patency. All biopsies were performed through the right IJV under sterile conditions. For the procedure, the right IJV was punctured by an $18 \mathrm{G}$ needle under ultrasound guidance. Then, a 0.035 inch $\mathrm{J}$ tipped guide-wire was introduced through the needle, and a $7 \mathrm{~F}$ short vascular sheath was placed into the IJV over the wire. A $5 \mathrm{~F}$ multipurpose catheter was inserted into the right hepatic vein through this sheath. Then, a venography was performed to confirm the catheter position. When the catheter position was satisfactory, a 0.035 inch super stiff Amplatz guide-wire (Boston Scientific) was placed into the right hepatic vein through the catheter. The biopsy set (Cook, Bloomington, IN) was sent to the right hepatic vein over the stiff guide wire. The biopsy site was adjusted with the use of an arrow placed on the outer part of the metallic cannula. The preferred biopsy site was the anterior part of the right hepatic 
vein, because the parenchyma at this site was more than in the other parts. Thereafter, biopsy was obtained with the use of an 18 gauge $60 \mathrm{~cm}$ Quick-Core biopsy needle (Figure 1). The number of needle passes was left to the experience of the radiologist who considered observation of the gross hepatic tissue specimen obtained. The number of needle passage times was noted. All the specimens were sent to the pathology unit in formalin solution for histopathological examination. The length and mean number of portal triads per sample of the material and the pathological diagnosis was noted. After completion of the procedure a control venography was performed for demonstration of acute complications. All the procedure related complications were noted.

The procedure was completed after removing the short vascular sheath. This was done while the patient was sitting and after removing the sheath, pressure was applied for five minutes.

All patients underwent postprocedural neck and abdomen ultrasound examination for the possible presence of hematoma and bleeding. Patients were kept in the hospital for 24 hours after the procedure. The clinical observation and abdominal palpation, control hemoglobin levels were followed up. Abdominal ultrasound examination was repeated when necessary.

\section{Results}

Technical success was achieved in 93 of 97 (95.8\%) patients. In two patients with Budd Chiari syndrome, hepatic veins could not be catheterized and opacified and in the remaining two patients, the hepatic veins could be catheterized with the stiff guide wire but the stiff cannula did not advance over the guidewire due to the acute angle between the IVC and hepatic veins (Figure 2). The mean number of needle passages required for each patient was $2.75 \pm 0.8$ (range=2-7). The sample lengths ranged from 1 to $22 \mathrm{~mm}$ (mean: $6.6 \mathrm{~mm}$ ). The mean number of portal triads per sample was 7.9 (range: 2-25). Histopathological diagnosis could be established in $98.9 \%$ (92 of 93) of the patients. In one patient, the specimen was lost in the pathology unit. Histopathological diagnosis was cirrhosis in 32 (34.7\%) patients, chronic hepatitis in 24 (26.1\%) patients, Wilson's disease in $1(1.1 \%)$ patient, hemochromatosis in 3 (3.3\%) patients, graft versus host disease in 7 (7.6\%) patients, primary biliary cirrhosis in $1(1.1 \%)$ patient, acute cholestatic hepatitis in $6(6.5 \%)$ patients, adenocarcinoma in 1 (1.1\%) patient, myeloproliferative infiltration in 2 (2.2\%) patients, hemosiderosis in $1(1.1 \%)$ patient, and the findings were normal in 14 (15.2\%) patients. Non-inflammatory steatosis was accepted as a normal finding. The histopathological diagnoses of TJLB are given in Table 1. Local hematoma at the puncture site was seen in 1 (1\%) patient. No additional complications were noted.

\section{Discussion}

Percutaneous biopsy is the preferred biopsy technique for a definite diagnosis of diffuse liver disease. However, when there is a contraindication for PLB, such as coagulation abnormalities, and thrombocytopenia, the mortality and morbidity of the procedure increase $(6,7)$. The most important complications for PLB are intraperitoneal bleeding and capsular perforation (8). Transjugular liver biopsy decreases these com-
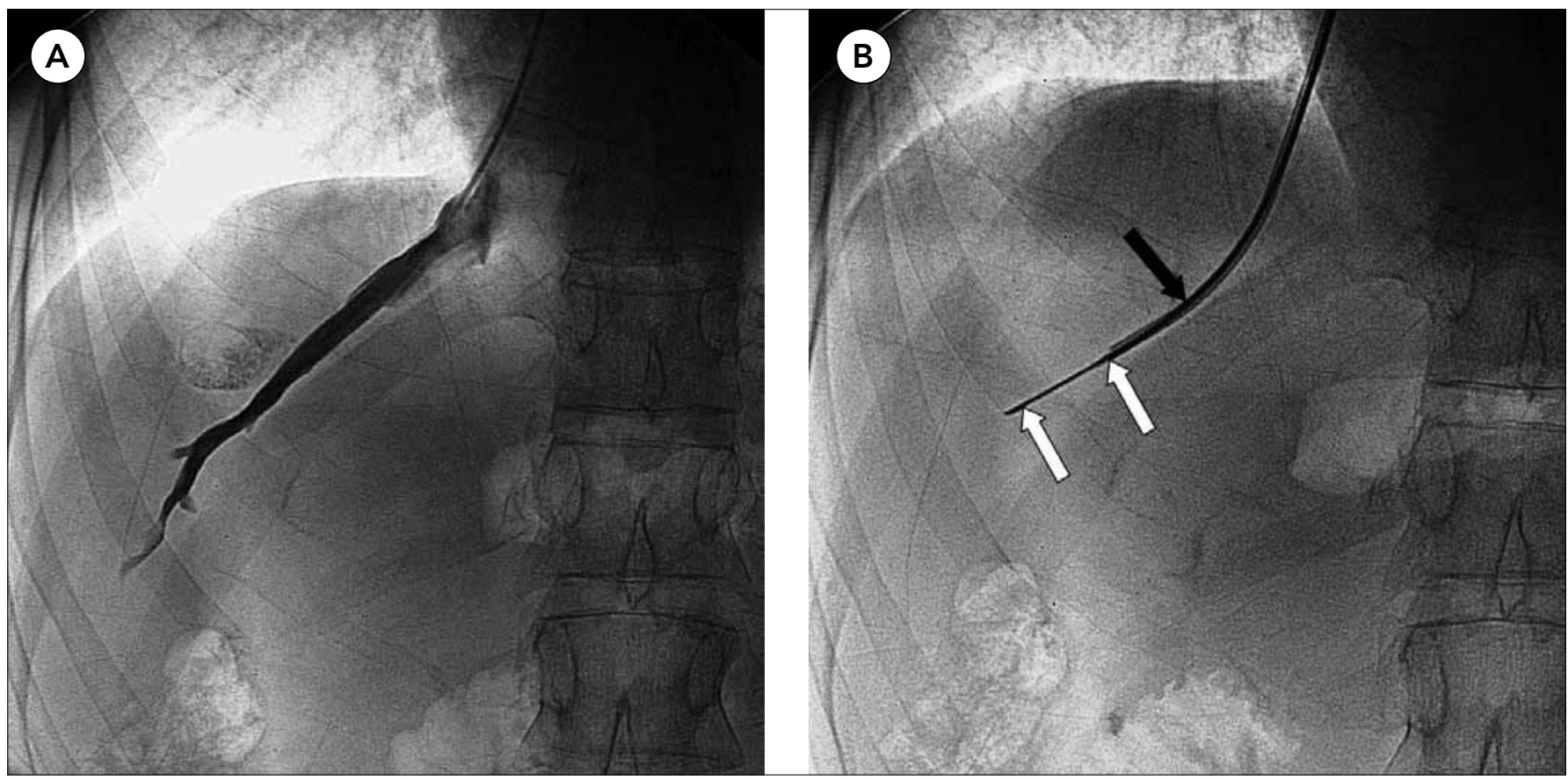

Figure 1. A 23-year-old male with presumptive clinical diagnosis of cirrhosis, with an international normalized ratio of 1.7. A) Venogram of right hepatic vein after catheter positioning into vein shows normal flow in vein with suitable angulations of vein to inferior vena cava. B) Image shows a 7-F stainless steel trocar (black arrow) in the right hepatic vein, with the semiautomated biopsy needle extended (white arrows) just before obtaining biopsy core specimen 


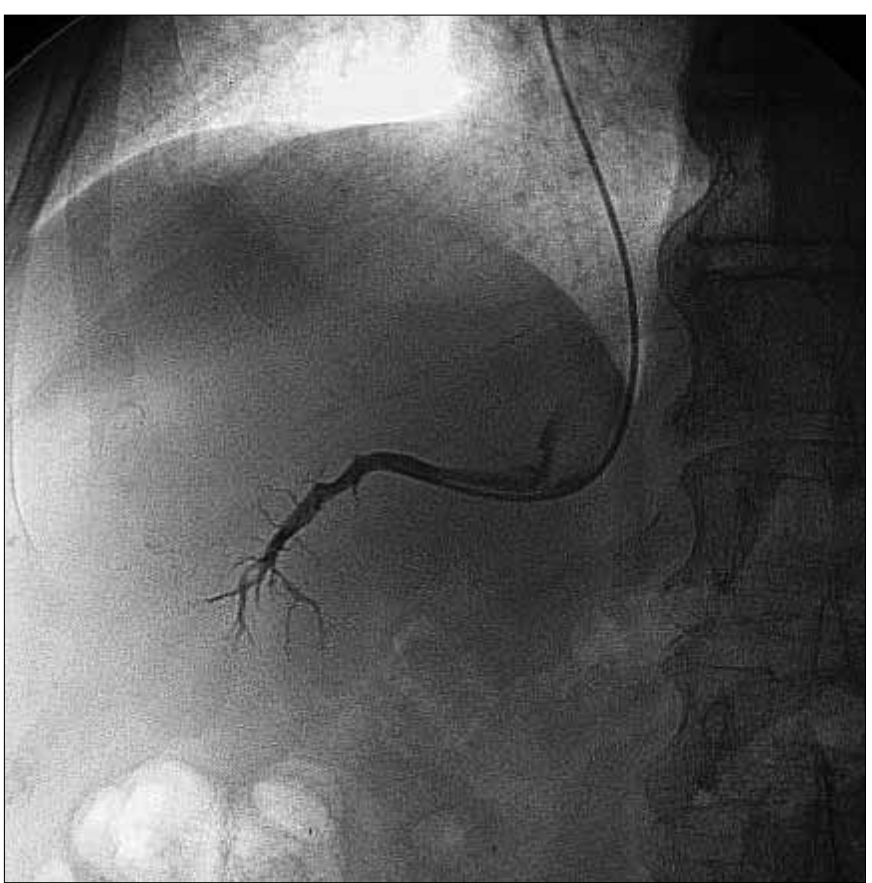

Figure 2. A 57-year-old male patient with an international normalized ratio of 1.8 . In this patient, the stiff cannula did not go over the guide-wire due to acute angle between inferior vena cava and hepatic vein

Table 1. Histopathological diagnosis of transjugular liver biopsy

\begin{tabular}{|lc|}
\hline Histopathological diagnosis & $\mathrm{n} / \%$ \\
\hline Cirrhosis & $32(34.7 \%)$ \\
Chronic hepatitis & $24(26.1 \%)$ \\
Wilson's disease & $1(1.1 \%)$ \\
Hemochromatosis & $3(3.3 \%)$ \\
Graft versus host disease & $7(7.6 \%)$ \\
Primary biliary cirrhosis & $1(1.1 \%)$ \\
Acute cholestatic hepatitis & $6(6.5 \%)$ \\
Adenocarcinoma & $1(1.1 \%)$ \\
Myeloproliferative infiltration & $2(2.2 \%)$ \\
Hemosiderosis & $1(1.1 \%)$ \\
Normal findings & $14(15.2 \%)$ \\
Total & 92 \\
\hline
\end{tabular}

plications since the capsule is not perforated. Obtaining the biopsy from the anterior part of the right hepatic vein, since the parenchyma is thicker here, may prevent capsule perforation and thereby reduce the complication rate. However, when the catheter is stuck in the liver parenchyma and a biopsy is obtained in this position, bleeding may occur. When a bleeding occurs, it may be detected during the procedure on control venography. The catheter should come as close as possible to the perforation site and gelfoam particles can be used to occlude the bleeding site (9). In case of hemophilia or hemoperitoneum after completing the procedure; blood, blood products, and fresh frozen plasma $(10-15 \mathrm{ml} / \mathrm{kg})$ can be used for treatment. When there is no clinical improvement, hepatic angiography and venography should be performed and the bleeding site should be embolized, if possible (10). There were no such complications in our study. Lebrec et al. (11) encountered one fatal intraperitoneal bleeding in 1033 TJLB. Kardache et al. (12) and Bruzzi et al. (13) did not encounter any complication. Little et al. (14) reported one asymptomatic liver perforation.

Insertion site hematomas and carotico-jugular fistulas are reported complications. Putting the sheet out, sitting the patient in an upright position decreases bleeding complications. However, when it occurs, compression is enough for treatment. When it persists then endovascular or surgical interventions may be used to treat these complications $(15,16)$. De Hoyos et al. (17) reported two severe hematomas and concluded that this was the result of not using ultrasound. We did not encounter any inadvertent puncture and related complications in this study, and we advocate that ultrasound should be used to decrease puncture site complications.

The preferred site for TJLB is the right IJV because this approach provides easy access to the right hepatic vein and thereby eliminates cardiac compression and vascular complications. Therefore we were able to use the right IJV in all 93 patients, but when the right IJV is tortuous or occluded, the left IJV or femoral vein can be used as the access site (18). However, the femoral approach needs a different biopsy set. We advocate that both biopsy set types should be present in angiography suites performing TJLB, because, we failed to get biopsy via jugular vein approach for two patients. If we had the femoral approach set we could have used it.

The most reported cause of technical failure in the literature, as in our study, was the inability of hepatic vein catheterization $(19,20)$. Chevallier et al. (21) altered the cannula angle of the metallic cannula manually in 8 of 139 patients to get better access by adjusting the IVC-hepatic vein angle. Another alternative for adjusting the IVC-hepatic vein angle is deep inspiration. When the patient inspirates deeply, the liver moves inferiorly and so a better IVC-hepatic vein angle may be achieved (9). We tried both manipulations for two patients but were not successfulin accessing the hepatic vein with the stiff cannula. Mammen et al. (9) used the transcaval approach in hepatic vein occlusion and IVC-hepatic vein angle discordance and they reported that this method was safe and effective.

Inability to puncture the IJV is the second common cause of technical failure (19). The use of ultrasound decreases inappropriate punctures and procedure time (22). Some authors have marked the IJV and accessed it through this marking, but with this method you may have inappropriate punctures even with minor movements of the patient (20). We used the "free hand technique" and all the punctures were without complications. In concordance with our study, Mammen et al. (9) reported $100 \%$ success for IJV puncture under ultrasound guidance.

Bruzzi et al. (13) reported that all the materials they obtained were sufficient for histopathological diagnosis. However, the reported rates of satisfactory material for histopathological diagnosis differ and the sufficiency of the specimen is decided with the number of portal spaces. More then 11 
portal spaces are classified as very good; $8-10$ is classified as good; 4-8 are near to optimal; less than 4 are insufficient. In another study 6-20 portal spaces are accepted to be sufficient, but even in this case it represents only $1 / 50.000$ of the liver (23). In our series, the mean number of portal triads in each tissue sample was 8 . Our results concur with those of Kardache et al. (12) (eight or more portal triads) and De Hoyos et al. (17) (6.24 \pm 3.18$)$.

The length of biopsy material is another parameter determining the quality of the specimen. When the length is more than $2 \mathrm{~cm}$, it is accepted as good biopsy material (24). In our study, the length of the material ranged from 1 to $22 \mathrm{~mm}$ (mean: $6.6 \mathrm{~mm}$ ). The size of tissue sample was thought to be small or limited, but a definitive diagnosis was reached in 92 of 93 patients. Evaluation of the size of liver specimen immediately after biopsy is very important. If the sample was not adequate for the diagnosis, a new biopsy should be performed in the same session, thus reducing discomfort and stress for the patient. Also, using this strategy reduces recurrent biopsy and uninterpretible results returned from the pathology. So we did not need to perform more than one biopsy for any patient later.

\section{Conclusion}

This study shows that TJLB is an effective and safe method for patients with coagulation abnormalities. The use of ultrasound for access site and knowledge of the maneuvers to get a better hepatico-caval angle may increase the technical success rate.

\section{Conflict of Interest}

No conflict of interest was declared by the authors.

\section{References}

1. Bravo AA, Sheth SG, Chopra S. Liver biopsy. N Engl J Med 2001;344:495-500. [CrossRef]

2. Dotter CT. Catheter biopsy. Experimental technique for transvenous liver biopsy. Radiology 1964;82:312-4.

3. Guido M, Rugge M. Liver biopsy sampling in chronic viral hepatitis. Semin Liver Dis 2004;24:89-97. [CrossRef]

4. Sheela H, Seela S, Caldwell C, Boyer JL, Jain D. Liver biopsy: evolving role in the new millenium. J Clin Gastroenterol 2005;39:603-10. [CrossRef]

5. Lebrec D. Various approaches to obtaining liver tissue-choosing the biopsy technique. J Hepatol 1996;25:20-4.

6. Zamcheck N, Klausenstock O. Medical progress: liver biopsy-the risk of needle biopsy. N Engl J Med 1953;249:1062-9. [CrossRef]

7. Velt PM, Choy OG, Shimkin PM, Link RJ. Transjugular liver biopsy in high-risk patients with hepatic disease. Radiology. 1984;153:91-3.

8. Piccinino F, Sagnelli E, Pasquale G, Giusti G. Complications following percutaneous liver biopsy: a multicentre retrospective study on 68, 276 biopsies. J Hepatol 1986;2:165-73. [CrossRef]
9. Mammen T, Keshava SN, Eapen CE, Raghuram L, Moses V, Gopi $\mathrm{K}$, et al. Transjugular liver biopsy: a retrospective analysis of 601 cases. J Vasc Interv Radiol 2008;19:351-8. [CrossRef]

10. Shulman HM, Gooley T, Dudley MD, Kofler T, Feldman R, Dwyer $D$, et al. Utility of transvenous liver biopsies and wedged hepatic venous pressure measurements in sixty marrow transplant recipients. Transplantation 1995;59:1015-22. [CrossRef]

11. Lebrec D, Goldfarb G, Degott C, Rueff B, Benhamou JP. Transvenous liver biopsy: an experience based on 1000 hepatic tissue samplings with this procedure. Gastroenterology 1982;83:338-40.

12. Kardache $M$, Soyer $P$, Boudiaf $M$, Cochand-Priollet B, Pelage JP, Rymer R. Transjugular liver biopsy with an automated device. Radiology 1997;204:369-72.

13. Bruzzi JF, O'Connell MJ, Thakore H, O'Keane C, Crowe J, Murray JG. Transjugular liver biopsy: assessment of safety and efficacy of the Quick-Core biopsy needle. Abdom Imaging 2002;27:711-5. [CrossRef]

14. Little AF, Zajko AB, Orons PD. Transjugular liver biopsy: prospective study in 43 patients with the Quick-Core biopsy needle. J Vasc Interv Radiol 1996;7:127-31. [CrossRef]

15. Duncan IC, Fourie PA. Percutaneous management of concomitant post-traumatic high vertebrovertebral and caroticojugular fistulas using balloons, coils, and a covered stent. J Endovasc Ther 2003;10:882-6. [CrossRef]

16. Ramsay DW, McAuliffe W. Traumatic pseudoaneurysm and high flow arteriovenous fistula involving internal jugular vein and common carotid artery. Treatment with covered stent and embolization. Australas Radiol 2003;47:177-80. [CrossRef]

17. De Hoyos A, Loredo ML, Martínez-Ríos MA, Gil MR, Kuri J, Cárdenas M. Transjugular liver biopsy in 52 patients with an automated Trucut-type needle. Dig Dis Sci 1999;44:177-80. [CrossRef]

18. Khosa F, McNulty JG, Hickey N, O'Brien P, Tobin A, Noonan N, et al. Transvenous liver biopsy via the femoral vein. Clin Radiol 2003;58:487-91. [CrossRef]

19. Kalambokis G, Manousou P, Vibhakorn S, Marelli L, Cholongitas $\mathrm{E}$, Senzolo $\mathrm{M}$, et al. Transjugular liver biopsy-indications, adequacy, quality of specimens, and complications. J Hepatol 2007;47:284-94. [CrossRef]

20. Soyer P, Fargeaudou $Y$, Boudiaf M, Rymer R. Transjugular liver biopsy using ultrasonographic guidance for jugular vein puncture and an automated device for hepatic tissue sampling: a retrospective analysis of 200 consecutive cases. Abdom Imaging 2008;33:627-32. [CrossRef]

21. Chevallier P, Dausse F, Berthier F, Saint-Paul MC, Denys A, Schny$\operatorname{der} P$, et al. Transjugular liver biopsy: prospective evaluation of the angle formed between the hepatic veins and the vena cava main axis and modification of a semi-automated biopsy device in cases of an unfavorable angle. Eur Radiol 2007;17:169-73. [CrossRef]

22. Soyer $P$, Lacheheb $D$, Levesque $M$. High-resolution sonographic guidance for transjugular liver biopsy. Abdom Imaging 1993;18:360-2. [CrossRef]

23. Sporea I, Sirli R, Popescu A, Cornianu M, Manciu C, Focsa M The quality of the fragment obtained by liver biopsy for staging chronic hepatitis. J Gastrointestin Liver Dis 2007;16:263-6.

24. Bedossa $P$, Dargère $D$, Paradis $V$. Sampling variability of liver fibrosis in chronic hepatitis C. Hepatology 2003;38:1449-57. [CrossRef] 